\title{
The Steep Spectrum Pulsar Population
}

\author{
D. L. Kaplan, J. M. Cordes \\ Cornell University, Space Sciences Bldg., Ithaca, NY 14853
}

\section{J. J. Condon}

NRAO, 520 Edgemont Road, Charlottesville, VA 22903

\begin{abstract}
We have sampled the population of compact $\left(\theta \leq 25^{\prime \prime}\right)$ radio sources with spectral indices $1.5 \leq \alpha \leq 3.2$ (where $S_{\nu} \propto \nu^{-\alpha}$ ) using two large scale radio surveys. This yields a statistically useful sample of 74 radio sources with $S_{1.4 \mathrm{GHz}} \geq 2.5 \mathrm{mJy}$ and $S_{365 \mathrm{MHz}} \geq 200 \mathrm{mJy}$. The majority of the sources appear to be extragalactic, resembling either high redshift radio galaxies or relic radio galaxies. However, six known pulsars were detected, as well as 16 sources of unknown nature that appear too small and have spectra too steep to be extragalactic. We are using VLBA, pulsar searching, optical/IR spectroscopy, and other techniques to definitively classify these sources. If any are new pulsars, they are potentially very interesting as they were missed by previous surveys.
\end{abstract}

Although many new pulsars have been found in recent years, almost all have been found as the result of standard period-dispersion searches. Such searches are limited in the parameter space they can explore. While appropriate for the majority of pulsars, they can miss pulsars that have unusually short periods, that are very highly dispersed, that are in very tight binary systems, or have very little intrinsic modulation (see e.g. Cordes \& Chernoff 1997). We therefore attempted to use the enormous amount of information present in large-scale radio surveys to find pulsars that had been missed previously. We did this by selecting all sources in the 1.4 GHz NRAO VLA Sky Survey (NVSS; Condon et al. 1998) and the $365 \mathrm{MHz}$ Texas survey (Douglas et al. 1996) with spectral index $\alpha \geq 1.5$ (where $S_{\nu} \propto \nu^{-\alpha}$ ). This is approximately the median spectral index for pulsars (Lorimer et al. 1995), but selects the $0.3 \%$ of general radio sources with the steepest spectra. We are above $90 \%$ complete for sources with $S_{365 \mathrm{MHz}} \geq 250 \mathrm{mJy}, 1.5 \leq \alpha \leq 3.2, \theta \leq 25^{\prime \prime}$, and $-35.5^{\circ} \leq \delta \leq 71.5^{\circ}$. For sample details, see Kaplan et al. (2000) and Kaplan, Cordes, \& Condon (2000).

Given the volume of sample space we wished to search, we believe that our criteria are reasonably good. Achieving a lower flux density limit would have meant sacrificing sky coverage. Relaxing the spectral index criterion would allow too many extragalactic sources into the sample. Applying additional criteria such as polarization, galactic latitude, or flux correlation could eliminate pulsars, given the range of observed properties (e.g. Xilouris et al. 1998 for polarization).

To futher classify the sources we obtained VLA A-array images at $1.4 \mathrm{GHz}$ and $5 \mathrm{GHz}$, with resolutions of 1.5 and 0.5 respectively. These observations, along with data from other catalogs, allow us to classify sources based on radio 
morphology and angular size. We found that of the 74 sources in the sample, 6 were known pulsars, 50 are clearly extragalactic, 2 have unknown morphologies, and 16 are unresolved (with $\theta \lesssim 00^{\prime \prime} 1$ ).

Of these sources, the ones of interest are the 6 known pulsars and the 16 unresolved sources. The unresolved sources appear superficially similar to the majority of the sources in the sample, in terms of their flux and spectral index distributions, their variability, and their galactic coordinates. However, their angular sizes do not agree with what one would expect for extragalactic sources of this nature (see O'Dea 1998). Sources with spectra this steep and sizes this small typically have spectral maxima at a few hundred $\mathrm{MHz}$; using data from other surveys we see no evidence of spectral maxima for our sources, often down to frequencies of $100 \mathrm{MHz}$ and below. These sources are therefore intriguing, and while the majority are probably not pulsars, a small number may be previously unknown pulsars. Regardless of number, all pulsars detected in this sample form a statistically homogeneous and useful sample, to which one can apply population analysis methods similar to those in Cordes \& Chernoff (1997).

In order to definitely classify these sources we are pursuing additional observations with a variety of techniques and wavelengths. Optical/IR imaging of those sources with counterparts (only $\sim 50 \%$; see Djorgovski et al. 2000 ) should allow us to determine spectroscopic redshifts, from which we could conclude whether the sources are extragalactic or not. We have also obtained but not yet reduced VLBA images of the most compact sources: if still unresolved by the VLBA the sources are almost certainly not extragalactic. Further observations, such as pulsar searches and improved radio spectra, are planned.

Acknowledgments. D. Kaplan was supported in part by NSF grant AST9530397 to Cornell University, NSF REU grants to the NRAO and the Arecibo Observatory, and the Fannie and John Hertz Foundation. 'The VLA and VLBA are operated by the NRAO, which is a facility of the National Science Foundation operated under cooperative agreement by Associated Universities, Inc.

\section{References}

Condon, J. J., Cotton, W. D., Greisen, E. W., Yin, Q. F., Perley, R. A., Taylor, G. B., Broderick, J. J. 1998, AJ, 115, 1693

Cordes, J. M., Chernoff, D. 1997, ApJ, 482, 971

Djorgovski, S. G., Bloom, J. S., Kaplan D. L., Cordes, J. M., Condon, J. J. 2000, in preparation

Douglas, J. N., Bash, F. N., Bozyan, F. A., Torrence, G. W., Wolfe, C. 1996, AJ 111,1945

Kaplan, D. L., Cordes, J. M., Condon, J. J. 2000, ApJS, accepted

Kaplan, D. L., Cordes, J. M., Condon, J. J., Djorgovski, S. G. 2000, ApJ, accepted

Lorimer, D. R., Yates, J. A., Lyne, A. G., Gould, D. M. 1995, MNRAS, 273, 411

O'Dea, C. 1998, PASP, 110, 493

Xilouris K.M., Kramer M., Jessner A., Hoensbroech A.v., Lorimer D., Wielebinski R., Wolszczan A. ApJ, 501, 856 